\title{
Tools for molecular risk-stratification for clinical purposes: CLL as a prototype
}

\author{
Daphne de Jong and Laura J van't Veer
}

\author{
Department of Pathology, The Netherlands Cancer Institute, Amsterdam, Netherlands \\ European Journal of Human Genetics (2004) 12, 423. doi:10.1038/sj.ejhg.5201220
} Chronic lymphocytic leukemia (B-CLL) has been recognized as a
disease with a highly variable prognosis. Several strong prognostic
markers to identify high-risk groups have been recognized over the past
few years and now justify risk-adapted stratification for treatment in
clinical trials. In a recent report, Schwaenen et $^{1} l^{1}$ describe a novel BAC-
array-based assay that allows rapid screening of genomic imbalances in
B-CLL that are associated with clinical outcome. ${ }^{1}$ This technique forms
a robust automated tool that meets the criteria for rapid implementa-
tion in clinical trials, as well as in daily practice in B-CLL and other
malignancies with dominant genomic imbalances that are associated
with prognosis.

Formerly, CLL was considered to be a uniform disease of the elderly, with an indolent clinical course that often did not require immediate treatment and that rarely was the direct cause of death for the patient. It is clear now, however, that CLL is a heterogeneous disease, both in terms of biological make-up and prognosis. Some patients with B-CLL survive for many years, not requiring any therapy, while others show rapidly progressive disease and die of their disease within months. Since the late 1970s, basic clinical parameters have been used as guidelines for treatment decisions. More recently, biological parameters have been found that can more reliably separate low- and high-risk patient groups. These include immunoglobulin variable heavy-chain gene (VH) mutation, cell-surface CD38 and intracellular ZAP70 expression and genomic aberrations. Low-risk B-CLL is characterized by mutated VH-genes and low CD38 and ZAP70 expression. High-risk B-CLL shows a reverse pattern of unmutated VH-genes and high expression of CD38 and ZAP70. ${ }^{2}$ There are several problems that may hamper implementation of these parameters in risk-stratification for daily practice. Primarily, standardization of scoring criteria and cutoff levels are a matter of ongoing discussion. ${ }^{3-8}$ Since, B-CLL is a very lowproliferative disease, the role of genomic aberrations has been mostly studied using fluorescent in situ hybridization analysis. Although specific DNA gains do occur, the disease is mainly characterized by DNA deletions. Most frequently, $11 \mathrm{q}, 6 \mathrm{q}, 13 \mathrm{q}$ and $17 \mathrm{p}$ deletions and trisomy $12 \mathrm{q}$ are found. Of these, $17 \mathrm{p}$ and $11 \mathrm{q}$ - rank as high-riskassociated aberrations and $13 \mathrm{q}$ - as single aberration as low risk. ${ }^{9}$

Although the relation between the different prognostic parameters and their relative prognostic impact may not be fully elucidated, it is clear enough that B-CLL can be separated into two distinct clinical subtypes. A strictly palliative approach no longer appears viable for all patients and risk-stratification for differential treatment seems justified. ${ }^{2}$ First attempts in clinical trials are currently being made both in Europe and in the US.

Validated reproducibility, low cost and preferentially high throughput should be considered as prerequisites for a diagnostic test to be implemented for clinical purposes. In their recent report in PNAS, Schwaenen et al ${ }^{1}$ describe a BAC/PAC-array-based tool for matrix- comparative genomic hybridization (matrix-CGH) that may meet these criteria. ${ }^{1}$ A genomic DNA microarray was developed, with 644 spotted clones derived from recurrently imbalanced genomic regions in B-CLL and encompassing 198 regions in 9-33 overlapping clones per site. Sensitivity and specificity of the microarray was tested in comparison with interphase cytogenetic data. Matrix-CGH showed 100\% overall sensitivity for B-CLL in cases with more than 53\% tumor cells with clonal aberrations in the sample. Sensitivity showed a slight drop when there were a lower proportion of clonally aberrant tumor cells in the sample. Matrix-CGH had a higher resolution and allowed detection of much smaller gained or lost regions. A specificity of $>99 \%$ was reached.

Provided that microarray facilities are available, matrix-CGH is an easy, rapid, and reliable method for screening genomic imbalances and is suitable for implementation in clinical trials and daily clinical practice. Development of disease-specific chips will allow wider use in clinical oncology. Alternatively, nonarray-based techniques as multiplex ligation-dependent probe amplification (MLPA) may be used. This PCR-based technique allows detection of genomic deletions and amplifications in over 100 loci. In a breast-cancer dedicated MLPA, a validated sensitivity and specificity of over $98 \%$ has been reached. ${ }^{10}$

Currently, treatment stratification on the basis of genomic alterations is operational in breast cancer and neuroblastoma. Breast cancer patients are selected for Herceptin treatment on the basis of Her2/neu amplification and neuroblastoma patients for intensified treatment on the basis of N-myc amplification. As more data become available on genomic prognostic markers, molecular stratification will become feasible in many areas of clinical oncology, increasing the demand for this type of validated molecular assay.

\footnotetext{
References

1 Schwaenen C et al : Proc Natl Acad Sci USA 2004; 101: 1039-1044.

2 Keating MJ et al: American Society of Hematology Educational Book, 2003, pp. $165-169$.

3 Rosenwald A et al : J Exp Med 2001; 194: 1639-1647.

4 Wiestner et al : Blood 2003; 101: 1944-4951.

5 Crespo M et al : N Engl J Med 2003; 348: 1764-1775.

6 Hamblin TJ, Davis Z, Gardiner A, Oscier DG, Stevenson FK: Blood 1999; 94 1848-1854.

7 Damle RN et al : Blood 1999; 94: 1840-1847.

8 Krober A et al : Blood 2002; 100: 1410-1416.

9 Dohner H et al : N Engl J Med 2000; 343: 1910-1916.

10 Hogervorst FB et al : Cancer Res 2003; 63: 1449-1453.

11 Ibrahim S et al : Blood 2003; 101: 4944-4951.

12 Hamblin TJ et al : Blood 2002; 99: 1023-1029.
} 